\title{
Aspects that contribute to the success of personalized Web applications
}

\author{
José Luís Reis ${ }^{1}$, João Álvaro Carvalho ${ }^{2}$ \\ ${ }^{1}$ ISMAI, Maia Institute of Higher Education, Research Unit UNICES, Maia, Portugal \\ ${ }^{2}$ Centro Algoritmi and DSI, Universidade do Minho, Guimarães, Portugal
}

\begin{abstract}
Personalized applications exhibit features adjusted to the preferences or needs of users and can provide benefits related to customer relationship or related to the efficiency of organizational work. This article presents a conceptual model that can be viewed as a contribution to the body of knowledge of Information Systems and Technologies professionals with responsibilities in the adoption, and development of personalized Web applications. These contributions include: a conceptual model that combines and articulates aspects that contribute to the success and to the quality of personalized information system (this model combines design features, facets that contribute to the quality and metrics that can be used to evaluate the performance of organizational work supported by personalized information systems). This set of aspects combines a synthesis of information so far scattered in the literature with proposals and recommendations with empirical knowledge based on an empirical study.
\end{abstract}

Keywords: Personalization, systems, information, quality, metrics

\section{Introduction}

With the globalization of information and communication technologies (ICT) (due mainly to wide dissemination of services based on Internet/Web), personalization become a reality in many Web sites and in some organizational information systems. The personalization of applications and computing platforms is a complex process. This work aims to address the following questions: a) what kind of factors contribute to the quality of a personalized information system? b) what aspects should be taken into consideration when implementing a personalized system? c) what factors influence the performance of a personalized information system?

This article presents the following research results: a) the main quality facets of a personalized information system; b) a conceptual model covering aspects such as the main features that contribute to the personalized system design, the quality facets and the metric for the work execution using personalized applications. 


\section{Personalization types}

There are several areas, both in academia and in organizations, where personalization is considered as an issue. Examples include: management, economics, marketing and sales, political science, sociology, anthropology, medical sciences, clinical information systems and computer science [1]. In what concerns information systems, the main goal to personalize ICT applications is to fulfill the needs and preferences of a person (or group of persons) that use a specific personalized ICT application.

Personalized ICT applications (also named as Personalized Applications - PA) facilitate the interaction among the various actors that cooperate within an organization, and the interaction with information providers and information recipients. It allows people to have easier access to content and services.

The "individuality" of consumer information is the key aspect in the personalization definition, implying that an individual's attributes, such as identity, preferences, constraints and provision (example, location and presence) have a potential impact on the personalization process [2].

According to the personalized features and purpose of a PA it is possible to establish different typologies to achieve personalization based on different aspects, including [1], [3]:

- What is personalized? The system characteristics that will be personalized (for example: content, user interface, information features, and channels for information delivery).

- Who are the personalization targets? Individuals or groups/categories of individuals.

- Who sets the personalization (user or system, or both)? Implicit or adaptive/proactive, if the personalization is done automatically by the system when the system, through collaborative filtering techniques, discovers product preferences of a customer. Explicit or reactive if individuals participate in providing information about their preferences and needs.

- How is personalization achieved? There are two possible ways to achieve personalization: a) through processes of personalization that not require intervention from users - observational [4] or implicit personalization; b) personalization processes that somehow require the participation of users. In the last case, when users are interrupted in the work processes they are involved in to provide answers to questions that are placed upon it, the personalization is said to be intrusive or explicit personalization [5].

\section{Quality facets of a personalized information system}

The quality of a system is measured by its desirable characteristics. For example, in Web enable e-commerce support systems, quality involves usability, trust/safety, reliability, adaptability and performance (response time) [6]. The quality of information refers to the comprehension of the system contents [7] and has characteristics like: form (more discursive or more graphical qualitatively or quantitatively, etc. depending on the technology), age (the time interval between the 
time the problem/event arises and the time when the user becomes aware of it), frequency (number of times information is available over a period of time), timeliness, relevance, security, profitability, cost, accuracy, completeness and consistency [8]. The individual impact of the system in the user can be measured by the system performance in terms of decision making, effectiveness of quality tasks.

In the case of a Web system, for having quality the system must be personalized, complete, relevant easy to obtain and memorize [6]. Service quality refers to the total support provided by the service provider, particularly in aspects related to the trust/assurance, empathy, responsiveness and accountability [7]. The evaluation of information quality, considered an economic asset, derives from the state of lack of need that the user is (what you want to meet to annul the uncertainty and the imbalances to be corrected) [9]. System personalization can improve the shape, age and frequency of information, tailored to the needs of the users [10].

The quality facets of a personalized system are from various types and have different characteristics. The following paragraphs present the quality facets of a personalized IS, mentioning their characteristics and underlying assumptions.

\section{Usability}

The software should be understood, learned, used and appreciated in a positive way by the user according to their personal characteristics, when used under specified conditions. It should be easy to use, must have a help system easy to understand, especially in a language understood by the user and must have good presentation according to user's preferences.

\section{Adaptability}

Ability to adapt to different user profiles according to their preferences and needs. Allow user to select their favorite colors, layout options, shortcuts for quick access to features, information and forms to submit, etc. It must be linked to suits of the system provider, as well as presents and expands needs of those who use the system according to the conditions specified by who provides the system.

\section{Flexibility}

Different interface forms allow the same system to be used in different circumstances and used by users with different characteristics, thus achieving the same system to achieve the same goal of several ways. A personalized system must recognize if the user is accessing it via a mobile system (smartphone) or via a fixed system (desktop) and allow the options and features are available in different size screens and graphics motion. The system must be prepared to be used according to preferences and context of use, a smartphone, a computer, a tablet, a PC, a multimedia kiosk, a PDA, a plasma media system, either with a touch screen, with or without a mouse, with a pen, in different operating systems, Windows, Android, Linux, Vista, Mac, etc. with different bandwidths, in any type of navigation system, in different languages, etc. always according to users preferences. To verify the consistency and flexibility in a system is guaranteed to make tests on the way with a minimum of clicks and how quickly one can achieve a certain goal, for example finding a product in an e-commerce shop.

\section{Ubiquity}

With digital identity, users can change their profile according the contexts and situations where they are, and it becomes even more complex to manage these processes to understand the link between the "real identity" and "digital identity" [11]. The information recorded in databases cannot define the texture of our lives leading to 
uncertainty about the information. The data, rather than providing a portrait of our personalities, are compiled and stored, as were captured, from what we did, not often have an underlying reason for having done, which means that we are reconstituted on the digital basis data as people made up of data - paradoxically the problems associated with violation of the privacy of personal data, which do not allow their use and dissemination, are distorting what we are in the digital world. As computers become increasingly pervasive (ubiquitous) in our lives, in smart environments that lead to greater collective intelligence, is need to create systems for identifying users who do control, with efficient and easier parts of information belonging to a particular individual which are available to others in the environment, with policies and protocols that require access to this information in an increasingly safe and enabling access to more than one platform [12]. For example, Google allows the same login and password for access to different applications and platforms (Gmail, YouTube, Blogspot, Picasa, Googledocs, etc.). The W3C has created a script (roadmap) which refers to the personalization elements that allow the integration of several features in ubiquitous environment with Web access to all interested [13].

\section{Reliability}

Ability to maintain the level of performance when used under specified conditions, allow recovery of failures in time. Ensure robustness for use in extreme conditions, such as a high number of users simultaneously, with greater difficulty of access, in case of breaking system allowing to return to work at the same point of break without information loss.

\section{Opportunity}

The information must be displayed when the user needs it, and may be reactive, preferably pro-active, but always going to meet the needs of the user without being too obtrusive. Should take into account user preferences when is using the system and in accordance with the user profile (recommendations or notifications system).

\section{Efficiency}

Operate with a level of performance required for the quantity of resources associated with it, when used according to specified conditions. Allow register and remove in time, the information that the user need. Present the information in a legible, accurate and in accordance with user expectations.

\section{Effectiveness}

Should be found a way to combine the interest of who provides the information and who receives it, creating value to the system and for who use it. Should be optimized the existing resources for those who produce information (sell, provide a service, etc.) and who receives it (buy, watch, etc.).

\section{Security}

The system must be prepared to perform secure transactions without violating the laws that protect organizations and individuals regarding the processing of information in a secure manner. The system must provide accurate and useful information that the user needs in a safe manner, without violating their privacy and in accordance with the user's profile, according to their preferences for the presentation of such information, either in time or in the medium. Should ensure interoperability between different systems and must be secure in the use and in the registration of transactions. A personalized system must identify who uses, ensure privacy, set the preferences to allow use to infer how to use, integrate different 
components from different systems in the same work environment, possess security certification (encryption, etc.).

\section{Accessibility}

The system shall be designed and constructed to be accessible regardless of the user's profile, their physical limitations and types and versions of hardware or software based that will be used. Accessibility is characterized by attributes that let you set up the information system is "accessible" to all citizens in all the fullness users to the content available. The visual and auditory forms of content should be available to users, as well legends to explain that allow describing the more complex visual content, such as charts or diagrams; this content can be converted into voice through adapters [14]. W3C allows, by inserting in the address http://validator.w3.org/ the site pages, to verify that the HTML code for a set of hosted pages, accessible from that address meets the quality standards of HTML for improving the usability and accessibility of Web pages with the subsequent benefits of these quality standards [15], [16]. If this occurs, the W3C returns a connection (link) that lets put a symbol that "certifies" the inherent quality and accessibility of these pages. A personalized system should have features that do not allow the exclusion of any user. Thus, despite the personalization information system to be independent of access is desirable that the information system having these qualities can be used by any person according to personal characteristics.

Performance

The system must be able to increase performance levels of user benefits for the organization and for individuals. Allow the user to have easier access to their favorite menus, to the most used applications and the data it needs to improve its performance. The system must allow the needs of users according to their profile, in a computing environment pleasant easy to use and making the information available useful. According to preferences and the right time, with the right way, the system helps the user to solve their problems by increasing the levels of user comfort. It is often said that a computer application should take no more than 3 seconds to respond. The system must be prepared to respond in a timely manner to requests and queries that are performed by users with information adapted to user's needs and preferences, and without additional waiting time caused by personalization of information or interfaces.

\section{Design features of personalized applications}

The success of a system can be evaluated through a number of aspects that influence how it is used. The system performance evaluation should be made taking into account that there are external system aspects, for example, the system is installed on a computer that has the minimum requirements to support the system, either for reasons related to low memory or disk available space, either by a problem related to the characteristics of a data network, which may not have enough bandwidth to support the transactions necessary for the proper performance of the system, or because the user is using the system in a wrong device media. 
The internal components can influence the performance of a system, because most systems are designed to be used by individuals who require these components to be considered when systems are developed. The internal components of the system, relate to the characteristics of the architecture (design) of computer applications, need attention and should be respected. These are aspects relate to specifications associated with the source code of software that was developed, including techniques used in the construction of the system and in its components in the construction on the following three levels [17]:

- Control of its structure with aspects related with modularity, complexity, cohesion and consistency of the components of the control flow.

- Structure of information with aspects related to the overall structure and types of data, the articulation between data (coupling) and the system, the consistency of the flow and type of data, the integrity and complexity.

- Code related typography for example: format, separating modules or spaces/blank lines.

\section{Metrics for work execution using personalized applications}

Although there are several possible metrics for the different aspects associated with the efficient and effective performance of a system, let us focus on metrics that somehow contribute to the performance on the work using a personalized system [7], [18]:

- Time spent to complete a task in the system. For example, buy an electronic product in a Web store; the system can provide a personalized list of recommendations and prevent the user from losing time on research and transactions subsequent to the acquisition process.

- Number of tasks that can be completed over a period of time if the system is able to identify the sequence of actions that a user makes to complete a given task.

- Number of commands used and completed in a given period of time and access to the commands available to the users in a sequence according their usage preferences.

- Time spent to find and interpret the information in the guide/user manual.

- Time system inactivity (idle time).

- Marking notes of interest. In the case of a Web site, personalization should allow the user 'bookmark' a page or other elements of interest and they remain available for future reference.

- Accessibility of the system. The system shall be designed and constructed to be accessible regardless of the user's profile, their physical limitations, and types and versions of hardware or software that will be used.

\section{Integrated model of personalized systems design}

Taking into account the relevant aspects that contribute to the performance, the quality and the success of a PA, it is possible to construct a conceptual model that integrates the quality of a personalized application (facets that contribute to quality), 
the personalized software application design (design features) and the performance of work execution using the PA (metrics used to express the performance) - see Fig. 1.

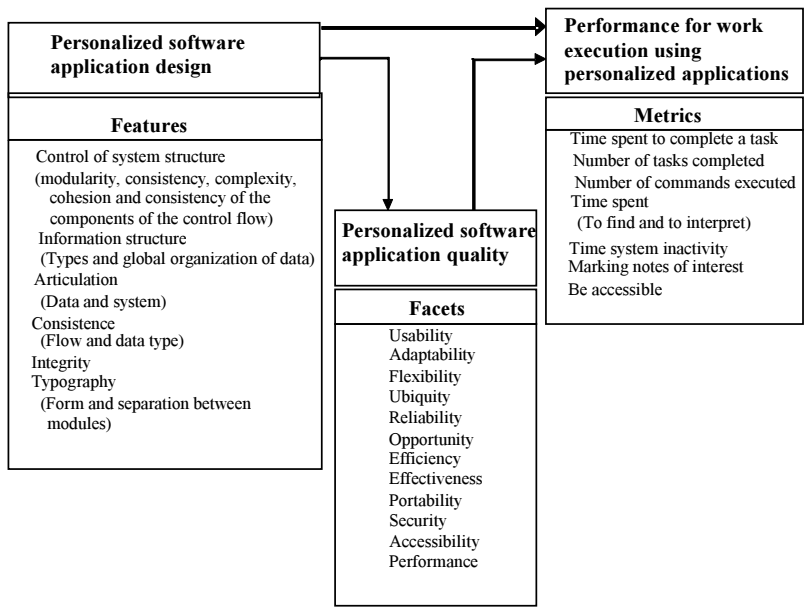

Fig. 1. Conceptual model with aspects that contribute to the design, quality and performance of a personalized system.

Fig. 1 depicts the existing relationship between design and quality control of the personalized system (for example, coordination between the control of system structure and the information structure should be organized in a way that ensures accessibility, usability, and adaptability to the users according to their needs). On the other hand, the facets of quality, such as usability and portability, are related to the performance of the work using the personalized system to ensure that the number and commands executed and the time spent on a task, regardless of location and the equipment that is to use, comply with acceptable run times. Furthermore, the design of a personalized system is related to the execution of organizational work system and must have acceptable performance.

\section{Case of practical application on CVRVV}

With the aim of validate the conceptual model with the aspects that contribute for the quality of a personalization, it is used the knowledge inherent in the development of personalized features on the system of Commission of Viticulture of the Region Vinho Verde (CVRVV), in its Extranet component. The system called INETSIV (SIV - Wine Information System) allows the institution's relationship with its business community and its municipal delegations. In this section of the work it is described CVRVV, the objectives of personalization in CVRVV, what was personalized and the aspects that contribute to the design, quality and performance of the CVRVV personalized system. 


\subsection{The CVRVV}

CVRVV is the entity responsible for the certification of the Portuguese wine products with designation of origin Vinho Verde and Regional Minho. The CVRVV is also responsible for safeguarding the assets of the Region and the institutional promotion in the markets. The Vinhos Verdes Region has about 25,000 producers who have an area of about $22,000 \mathrm{Ha}$ of vines, with 600 entities authorized to bottle on average 55 million liters of wine per year certificate in about 1000 trademarks. CVRVV organic structure sets seven working departments with about 60 employees. The departments ensure the normal functioning of the institution, ensuring certification of wine products, protection of heritage designation and institutional promotion of products and brands from Vinho Verde and Regional Minho. There are various software applications to automate the processes involved in certification, with a set of modules which correspond to different applications required for products certification management. The system contains a set of interfaces, including an Intranet, that enable the interaction between about 50 internal users and the organization and a Extranet accessible to approximately 600 Economic Agents as well as 45 municipal delegations.

\subsection{Objectives of personalization in CVRVV}

The project called Web Personalization in CVRVV developed a set of features to improve the way users (Economic Agents and Delegations) interact with CVRVV through the information system. The personalization project allowed to implement a set of personalized features, including:

- A system that records how the Economic Agents (EA) accesses different options via the Extranet (INETSIV), noting in particular the selected options, time of use, as well transactions each done. This system allows users to have access to a range of information related how each user uses the different options available on the Extranet. - System access options available through facilitating elements, including through quick access buttons to the computer applications system.

- System which allows registration and access to e-mail messages that are personalized according the EA profile.

- Customization of interfaces brands of products with designation of origin certified by CVRVV in its component Extranet, Intranet and Internet. This system component requires an explicit action by EA to change the information associated with their brands.

The personalized features of INETSIV are: 1) personalized interfaces for Economic Agents and Delegations, creating personalized quick access buttons, 2) personalized the messages on the main screen with useful and relevant information to users, 3) customize the mail box; 4) customize interfaces brands. It was also created personalized information elements, which allow access to statistical information from the users. To implement these personalized features where adapted personalization implicit types in most of the functionality's, with the exception of customizing interfaces of the brands and the selection preferences regarding quick access buttons, which require user intervention with explicit personalization. 


\subsection{Aspects that contribute to the design, quality and performance of the CVRVV personalized system}

The responsibility on the aspects that contribute to the success of the performance of the information system is from the project coordinator, and even if these aspects are already implemented in the system in operation, should be tailored to the personalized features. Should be implemented, as much as possible, the criteria that influence the facets that contribute to the quality of PA (functionality, reliability, usability, maintainability, adaptability, timeliness, effectiveness, productivity, satisfaction, shape and frequency).

As regards the facets that contribute to the quality of the personalized system in CVRVV the most relevant are contained in the system in operation, namely:

- Functionality's appropriated to regulations required by law for certification of products in the wine sector, according to the rules that articulate the specific needs of each user (CVRVV manage a very heterogeneous universe of associates). It is also guaranteed interoperability among information systems, particularly with an external entity (Institute of Vine and Wine) at the level of information that registration is available to members who use the system, as well as some associated with larger (are they are able to make investments in IST) that have information systems that communicate with the CVRVV in an automated way (without human intervention) to make certain transactions (for example participation to affix seals of certification of products in the bottles). In the system of CVRVV there is a guarantee of identifying who uses the system in a safe, secured either by rules imposed by the internal CVRVV system's or by a company that guarantees the safety certification, which ensures the privacy of transactions. The PA in CVRVV also allows users to define their preferences on the options associated with quick access buttons, while also infer, from the use, which the most used options, presenting them in these default buttons.

- Reliability that keeps the level of performance under different system access (a user who has access the system through reduced bandwidths has no problem accessing the CVRVV system). When a transaction is broken for any reason, the system allows recovering (in some cases) the transaction that was being made. The PA has no CVRVV user limit and even in extreme conditions with high numbers of users in real time (e.g. from electronic receipt of statements of harvesting and production, because the legislation requires to be between 1 October and November 15 th, rare are the days when you are not at all times working, virtually all delegations related to CVRVV as well as many of the members).

- The usability of the information system is appreciated by the user's majority. The available software is easily understood by users, and it is not necessary specific training to promote the system because it is easy to use.

- The efficiency is guaranteed by the good performance of the system that allows recording and presenting timely information according the user needs, presenting them in a legible, accurate and in accordance with the legislation (is there detail information about the registration of vineyards, which have some complexity, sometimes it becomes difficult to understand by users and the system help the user).

- The maintenance of CVRVV software applications is ensured in accordance with the requirements set by the IST managers, and new applications requested by departments or by CVRVV Economic Agent users (the personalized functionalities 
developed, together with the brand customization, arose from a system user need). The implementation of new features in the system, does not prevent the system is kept operating in a stable manner.

- The system portability is guaranteed, including the possibility of operating in different navigation systems (browsers), on systems with different bandwidths. However, not all features are prepared to function optimally in all touch screens.

- The adaptability of the system enables different profiles may be assigned in accordance with the characteristics of each Economic Agent, however it is not possible color selection, nor the provision of options horizontally or vertically (although the system is prepared for accept this preference is not yet implemented), the quick access buttons allow shortcuts for faster access to applications, but not yet implemented shortcuts access the screens applications, although the system provide this functionality.

- The opportunity in the information presentation at the time the user needs is ensured in most situations, however, the notification system is the same for all users, only differentiated by the type of Economic Agents rather than their individual profile.

- The system effectiveness is guaranteed by the way it can combine the interests of Economic Agents and CVRVV delegations. However, is needed more investments so that they can implement more personalized features.

- Higher the productivity, higher is the user satisfaction and higher is the satisfaction of the system provider. The quick access buttons, custom messages and the customized brands contribute to the better CVRVV information system productivity.

As regards the aspects that contribute to the design, quality and performance of a PA in the case of the system of CVRVV, there linkage between data and system structure information which are arranged in a way that ensures accessibility and usability that guaranteed articulation between usability and portability which allows better performance in the execution the system jobs. For example, it is guaranteed the number of command executed and the time spent on a task is always the same, regardless of where you are and the user equipment that is being used. Moreover, the design of PA is hinged to the performance of organizational job execution. For example, the time spent in execution of a task it's not superior to the established in the specifications because they create personalized information, because they are not hard processing, or the time taken to locate a specific change or information is superior to the times considered reasonable, thus the efficiency and effectiveness of the system of CVRVV are at reasonable levels.

\section{Recommendations}

The implementation of a personalized system on the Web goes through several stages. It is necessary to ensure the existence of certain conditions for the development, including: evaluating the business model and organizational processes that allow personalization, define personalization types according to the targets, analyze the coordination and combination of the facets that contribute to quality of a PA with the 
design features of the PA with the metrics used to evaluate the performance of organizational work and define the appropriate technologies to achieve personalization.

The project coordinator must take into account the quality aspects that contribute to the success of the information system. If these aspects have already been taken into account in the system in operation, they must be reviewed and adapted to new personalized functionality's. To minimize the risk of failure it is vital to assure the existence of the resources required to implement the facets that are vital to the quality of the personalized system (functionality, reliability, usability, maintainability, adaptability, portability, efficiency, effectiveness and productivity).

\section{Conclusions}

The conceptual model presented in this article corresponds to a synthesis of knowledge about the aspects that contribute to the quality of a PA that so far was dispersed in several sources. This dispersion is understandable to the extent that such knowledge results from R\&D work in different areas, each focusing on particular aspects of personalization. In this article there is no reference to the technologies that enable personalization, to interface devices and to ways of implementing personalization (personalization levels and dimensions). However the conceptual model provides a basis to sustain the improvement of business management activities. Personal computing and personal Web is a trend that will consolidate in the coming years. Knowledge about the users will increasingly be applied to personalize information content and forms of ICT usage, adjusted to the work environment, allowing users to do the configuration and content reorganization of their work environment to support their professional, personal and even activities related to training according to their preferences. Despite the limitations of this work, particularly relating to validation based on a single study, the conceptual model constitutes a stable base for PA projects and can be viewed as a contribution to the body of knowledge of managers and IST professionals involved in decisions regarding the adoption of personalized ICT applications.

\section{References}

1. Fan, H., \& Poole, M. S. What Is Personalization? Perspectives on the Design and Implementation of Personalization in Information Systems Journal of Organizational Computing and Electronic Commerce, 16(3-5). pag 179-202. (2006)

2. Toth, K., \& Nagboth, S. R. Constraint-Based Personalization Model: Multi-Channel Messaging. Oregon, USA: Dept. of Computer Science, Oregon State University. (2002)

3. Anand, S. S., \& Mobasher, B. Intelligent Techniques for Web Personalization. Springer, USA. (2005)

4. Mulvenna M., A. S. Personalization on the Net using Web Mining. 43(8), 122-125. Communications of the ACM. (2000). 
5. Perugini, S., \& Ramakrishnan, N. Personalizing Interactions with Information Systems. Blacksburg - Virginia: Department of Computer Science. (2002)

6. Delone, W., \& McLean, E. The DeLone and McLean Model of Information Systems Success: A Ten-Year Update. (2003)

7. ISO, I. S.. Ergonomics of human-system interaction - Usability methods supporting humancentred design. ISO/TR 16982. (2002)

8. Oliveira, A.. Anatomia e metabolismo do processo decisório em contexto empresarial * Algumas Questões. Ciências Económicas e Sociais. (1999).

9. Oliveira, A. Análise do investimento em sistemas e tecnologias da informação e da comunicação. Lisboa: Sílabo. (2004).

10. Mobasher, B., Cooley, R., \& Srivastava, J. Automatic Personalization Based on Web Usage Mining. Obtained in 09.10.2012, de DePaul University - College of Computing and Digital Madia: http://maya.cs.depaul.edu/ mobasher/personalization/ (2000)

11. Black, J., Downes, K., Field, F., Mozdzanowska, A., \& Steel, K. Technologies:, The Metaphors of Emerging Unpacking the disconnects between the "what" and the "how" in the world of "online shopping". (M. E. Technologies, Ed.). (2006)

12. Cutting, D. Identity Management in Context-Aware Intelligent Environments . University of Sydney, School of Information Technology. (2006)

13. Heath, A., \& Schwerdtfeger, R. W3C Personalization Roadmap: Ubiquitous Web Integration of AccessForAll 1.0. Obtained in 24.09.2010, from W3C: http://www.w3.org/TR/UWA-personalization-roadmap/ (2009).

14. Henry, S. L. WAI Resources on Introducing Web Accessibility. (W3C) Obtained in 24.09.2011 from Web Accessibility Initiative (WAI): http://www.w3.org/WAI/gettingstarted/Overview.html (2005)

15. Arch, A. Ooutdated Incompleted Draft of Auxiliary Benefits of Accessible Web Design. (W3C) Obtained in 04.07.2012, from W3C - Web Accessibility Iniciative: http://www.w3.org/WAI/bcase/benefits.html\#intro. (2002)

16. Dubost, K. My Web site is standard! And yours? Obtained in 02.06.2012, from W3C Quality Assurance: http://www.w3.org/QA/2002/04/WebQuality.html\#Standard (2003).

17. Oman, P., \& Hagemeister, J. Metrics for Assessing a Software Systems Maintainability. (IEEE, Ed.) Conference Publications on Software Maintenance. Pag. 337-344. (1992)

18. Pereira, C., \& Capeto, R. Indicadores para avaliação de websites. Obtained in 15.12.2011, TeleCentros: http://www.telecentros.org/discapacitados/parametro=9861\&idioma $=$ br.html (2002) 